\title{
Determination of Therapeutic Values of Düzce/Yığılca Honeys by Underlining Overlooked Parameters
}

\author{
Meral Kekeçoğlu ${ }^{1,4, a, *}$, Tuğçe Çaprazlı ${ }^{2, b}$, Emel Çalışkan ${ }^{3, c}$, Serpil Uğraşs,d \\ ${ }^{1}$ Department of Biology, Faculty of Art and Science, Düzce University, 81620 Düzce, Turkey. \\ ${ }^{2}$ Düzce University, Düzce Vocational School, Beekeeping Program, 81620 Düzce, Turkey \\ ${ }^{3}$ Department of Microbiology, Faculty of Medicine, Düzce University, 81620 Düzce, Turkey. \\ ${ }^{4}$ Development and Application Center, Düzce University Beekeeping Research, 81620 Düzce, Turkey.
}

*Corresponding author

A R T I C L E IN F O A B S T R A C T

Research Article

In this study, we aimed to determine the therapeutic value of local Yı̆̆ 1 lca honeys by examining their physicochemical characteristics, phenolic content, radical scavenging activity, and antimicrobial properties by underlining some overlooked parameters. Honey samples ware divided into two main groups as $\mathrm{CH}$ and $\mathrm{FH}$ based on melissopalynologycal analysis. Antimicrobial activity

Received : 06/11/2021

Accepted : 23/12/2021 of collected honey samples were investigated on important hospital-acquired infections bacteria strains; MRSA (Methicillin-resistant Staphylococcus aureus), MSSA (Methicillin-sensitive Staphylococcus aureus), Escherichia coli, and Acinetobacter baumannii. Although CH's antimicrobial activities were found slightly higher than the FH's, there isn't statistically significant differences between two type honeys. However, the antioxidant activity of $\mathrm{CH}$ was found significantly higher than FH. Surprisingly, a higher amount of isomaltose was determined in addition to the total phenolic content in $\mathrm{CH}$ compared to $\mathrm{FH}$. There has been found positive correlation between isomaltose amount and zone diameters for MRSA and A. baumannii. We would like to draw attention to isomaltose for its health benefit structure since these parameters may be influence honey's therapeutic value. We recommend that isomaltose and invertase enzyme should be included in to the honey codex standards suitably depending on the monofloral and multifloral honey's specific structure, to sensitively standardize and control their quality and therapeutic value. Our data revealed a positive correlation between antioxidant and antimicrobial activity and total phenolic content and higher isomaltose amount.

Antimicrobial

Antioxidant

Chestnut honey

Flower honey

Invertase http://orcid.org/0000-0001-9109-0969 http://orcid.org/0000-0002-1867-5781 (i) http://orcid.org/0000-0002-2564-8343 iD http://orcid.org/0000-0003-3444-1579
- tcaprazli@gmail.com serpilugras@duzce.edu.tr

This work is licensed under Creative Commons Attribution 4.0 International License

\section{Introduction}

The special features of honey were recognized by our ancestors, and it has an important place among human nutrients since then. According to the earliest rock drawings in Bicorp near Valencia in eastern Spain, the honey collection has an ancient, 8000-year-old history and having been consumed during the Prehistoric Periods (Crane, 1997). In addition to its historical nutritional use, honey also has been applied as a treatment for burns, skin diseases, stomach ailments, etc. (Kuropatnicki, et al., 2018; Özkök et al., 2018).

Turkey has a varied climate diversity incurred by its special geographical position and features. This situation has led to the existence of a variety of plant forms such as forests, shrubs or bushes, and grasses in the natural vegetation (Günal, 2013). Owing to its phytogeographical structure, Turkey is quite rich in unique floral sources to produce a great variety of monofloral honeys (sunflower, chestnut, astragalus, cotton honeys, etc.). Chestnut honey is one of the high-quality monofloral honeys from Turkey and it is mostly produced on the north-facing slopes of the Black Sea region, where the humid-temperate climate can meet the needs of the Castanea sativa trees. Due to its antioxidant properties, dark color, and unique flavor, it has been attracted the attention of customers in the domestic market (Ömür, 2015; Günal, 2013; Çöl Ayvaz et al., 2018).

Plant diversity has a direct effect on honey, and this varies mainly according to geographic location, sources, and climate zone. Its phytogeographical origin makes honey a unique and valuable product. The pollen and nectar of each plant around the hive have their own special 
ingredients and when processed with honeybee enzymes such as invertase, diastase, glucose oxidase and glucosidase, they converted to honey (Valentini, et al., 2010; Chauhan, et al., 2017).

The pollen content contributes to the phenolic component of the honey. The phenolic component is one of the reasons for the antioxidant activity of honey since the antioxidant substances contain molecules that delay cellular damage and inhibit oxidative stress and free radical reactions that could cause many diseases when the body exceeds a certain ratio (Frankel, et al.,1998; Taormina, et al., 2001; Young and Woodside, 2001; Beretta et al., 2005; Holderna-Kedzia and Kedzia, 2006; Bertoncelj et al., 2007; Lobo et al., 2010; Liu et al., 2012; Cimpoiu et al., 2013; Moniruzzaman et al., 2014; Nayik and Nanda, 2015; Nimse and Palb, 2015; Boussaid et al., 2018; Çöl Ayvaz et al., 2018). The increasing bacterial resistance to antibiotics has become an important problem in recent days. For example, semi-synthetic penicillin resistance has developed due to the $\beta$-lactamase formation of Staphylococcus aureus strains, which are one of the major causes of the wound and burn infections. Encountering these findings raises the need for the development of new antibiotics. To solve this problem, scientists began to focus on active natural antimicrobial resources such as honey. The antimicrobial property of honey has been known since ancient times. To determine honey's antimicrobial effect, in vitro studies carried out by using S. aureus, E. coli, Acinetobacter spp. and many other bacteria strains. And these studies determined that honey inhibits these bacteria strains. However, different inhibition zone results were obtained according to the floral sources and different structure of the honey samples (Bravo et al., 1998; Basualdo et al. 2007; Estevinho et al., 2008; Almasaudi et al., 2017). Escherichia coli is a Gram-negative bacterium with a wide range of antibiotic resistance mechanisms, particularly the extended-spectrum beta-lactamase and AmpC type beta-lactamase, which can be encountered in many infections, especially urinary tract infections (Bayram, et al., 2012). Acinetobacter baumannii is also an important Gram-negative bacterium for which a new antibiotic should be developed as it is a frequent cause of hospital infections and multiple-drug resistance (Al Bshabshe et al., 2016).

There is plenty of ingredient which providing the antimicrobial properties of honey and all these ingredients are directly and indirectly related to each other. Lack of an enzyme in honey may cause changes in the ratio of substances in honey and a decrease in its quality. For this reason, all substances in honey should be carefully examined and should not be ignored. One of them is sugar profile. When we examined the possible antimicrobial activities of honey sugars, isomaltose caught our attention for its biodegradability, antimicrobial activity, non-toxic structure, usage in foods as a low-calorie sweetener, and boosting of intestinal microflora, as well as its being safely categorized as a prebiotic, precursor metabolite. It has also been found to improve the well-being of humans and animals when ingested orally on a daily dosage basis (Lee et al., 2008; Gerschenson et al., 2017; Fatoki et al., 2018). The other ingredient is Invertase enzyme which is one of the most active enzymes found in the natural compound of honey. Invertase catalyzes the hydrolysis of sucrose into fructose and glucose (Derebaşı et al., 2014). Invertase activity is generally sensitive to storage conditions, and due to this sensitivity, it has been used as a freshness indicator. Hence, the invertase enzyme is more sensitive to heat than diastase enzyme and it is considered as a better indicator of freshness than diastase (Oddo, et al., 1999; Machado DeMelo and Almeida-Muradian, 2018). Although invertase is a sensitive heat indicator, it is not included in the Codex standards and Turkish Food Codex although it has been used as a honey standard by beekeeper associations in Belgium, Spain, and Germany and also by private companies (Bogdanov et al., 1999). It has been stated that the invertase value of fresh and untreated honey should be higher than 10 (Duisberg and Hadorn, 1966; Bogdanov et al., 1999; Oddo, Piazza and Pulcini, 1999).

As stated before, all ingredients vary depending on phytogeographical origin and they are bound by a natural balance, thus affecting the therapeutic value (antimicrobial, antioxidant, phenolic content) and physicochemical properties of the honey. As stated before, all ingredients vary depending on phytogeographical origin, and they are bound to each other by a natural balance, also they are affecting the therapeutic value (antimicrobial, antioxidant, phenolic content) and physicochemical properties of the honey. Therefore, through comprehensive studies should be possible to base these monofloral honeys on their phytogeographical origin and to determine which honey is useful for each specific disease. Our aim in this study; to compare and clarify the therapeutic value through physicochemical properties and antimicrobial activities of chestnut and flower honey produced in Turkey and to emphasize important overlooked parameters for determining the therapeutic value and quality, such as Isomaltose and Invertase enzyme content.

\section{Materials and Methods}

\section{Honey Samples}

Twenty-three honey samples were collected from local beekeepers of Yığılca district of Düzce city in Western Black Sea of Turkey. Honey samples were kept in sterile glass jars and stored at $20^{\circ} \mathrm{C}$ in the dark pending analysis.

\section{Melissopalynologycal Analysis}

Microscopic analyses were performed using the internationally recognized method (Maurizio, 1951; Louveaux, et al., 1970) modified by Kambur, et al. (2015).

After the pollen analysis, the pollen counts percentage of each sample were determined as dominant pollen $(\geq 45 \%$ ). Since chestnut honey was overrepresented in the monofloral honey category (chestnut pollen concentration $>70 \%$ ), we divided the honey samples into two main groups as chestnut honey and flower honey (Terrab et al., 2003; Turkish Food Codex, 2020).

\section{Physicochemical Analysis}

The moisture content of the honey was determined based on the AOAC 969.38 method, using the Lega also Refractometer HB90. A pH meter VWR pH $1000 \mathrm{~L}$ was used to measure the $\mathrm{pH}$ of a $75 \mathrm{ml}$ solution of $10 \mathrm{~g}$ honey prepared in Milli-Q water (Millipore Corporation, Billerica, Massachusetts, USA) (Bogdanov, 2009). 
Hydroxymetilfurfural (HMF) was made according to the IHC (2009) method adapted from the Winkler (1955). Invertase and proline content analyses were also made according to IHC (2009). The absorbance of the solutions was measured at 400 and $510 \mathrm{~nm}$ using a UV-vis spectrophotometer (VWR spectrophotometer UV$3100 \mathrm{pc}$ ). Diastase activity was analyzed according to the modified TS 3036:2002. Honey ( $5 \mathrm{~g}$ ) was poured into a 50 $\mathrm{mL}$ beaker and dissolved by adding 30-40 were $\mathrm{mL}$ of water and then transferred to a $50 \mathrm{~mL}$ flask to which distilled water was added to make up $50 \mathrm{~mL}$. A total of 57 separate incubation mixtures were used and the resulting honey-pure water-starch buffer mixtures were prepared. These were kept in a $48^{\circ} \mathrm{C}$ hot-water bath for $1 \mathrm{~h}$, after which $40 \mu \mathrm{L}$ of $0.1 \mathrm{~N}$ iodine solution was added to the test tubes and mixed. The first tube showing light blue was taken as the limit and the number of diastases corresponding to this test tube was read and saved digitally after the last point. The sugar profile was determined by high-pressure liquid chromatography (VWR-Hitachi Chromaster HPLC-RID) based on the method DIN 10758.

\section{Total Phenolic Content}

The total phenolic content of the 24 different honey samples was determined quantitatively using Folin Ciocalteu reagent (Chua et al., 2013). A gallic acid solution prepared with $\mathrm{dH}_{2} \mathrm{O}$ at a ratio of 1: 1 was used as the standard. The results of the reaction to the Folin-Ciocalteu reagent of six different concentrations (ranging from 10 to $500 \mu \mathrm{g} / \mu \mathrm{L}$ ) of the standard solution were evaluated by spectrophotometric measurement at $765 \mathrm{~nm}$ as performed by Chua et al. (2013). The graph was then drawn of the equation between the absorbance values and the concentrations. Similarly, the absorbance values of the 23 different honey samples were measured and the results were evaluated using the standard (gallic acid) graph (Chua et al., 2013).

\section{Radical Scavenging Activity}

The DPPH (2,2-diphenyl-1-picrilhydrazil) radical scavenging activity was determined using the DPPH free radical (Brand-Williams, et al., 1995). A methanol solution of freshly prepared DPPH radical was used in the study, and $1500 \mu \mathrm{L}$ of this solution was added to $750 \mu \mathrm{L}$ honey samples prepared at $1-100 \mathrm{mg} / \mathrm{mL}$ concentrations. The absorbance values at $30 \mathrm{~min}$ at $517 \mathrm{~nm}$ were measured on the spectrophotometer. Butyl hydroxy anisole (BHA) was used as the positive control (standard). A mixture of 750 $\mu \mathrm{L}$ methanol and $1500 \mu \mathrm{L}$ DPPH was used as the negative control and methanol was used as the blank (Chua et al., 2013). The experiments were repeated twice. The DPPH radical scavenging activity was calculated using the formula below. In addition, the half maximal inhibitory concentration (IC50) values were determined for the honey samples.

DPPH Radical Scavenging Activity $=[(\mathrm{A} 0-\mathrm{A} 1) / \mathrm{A} 0] \times 100$

Here,

$\mathrm{A} 0=$ Absorbance value of the negative control

A1= Absorbance value of the standard and the sample (Chua et al., 2013).

\section{Antimicrobial Activity}

The methicillin-sensitive Staphylococcus aureus (MSSA), Methicillin-resistant Staphylococcus aureus (MRSA), Escherichia coli, and Acinetobacter baumannii strains were isolated from various patient samples obtained from the Düzce University Medical Microbiology Laboratory.

Before each trial, fresh passages of the strains were grown on blood agar (Oxoid, England). The sensitivity of the honey samples and comparative antibiotics was determined via the disc diffusion method. The bacterial suspensions prepared in $0.5 \mathrm{McFarland}$ turbidity standards were spread on the surface of Mueller Hinton agar (Oxoid, England). After incubation at $37^{\circ} \mathrm{C}$ for $24 \mathrm{~h}$, the zone diameters were measured and evaluated (Rani, Budumuru and Bandaru, 2017).

Antibiotic discs (Oxoid, UK) for the antibiotic's penicillin $(1 \mu \mathrm{g})$, cefoxitin $(30 \mu \mathrm{g})$, erythromycin $(15 \mu \mathrm{g})$, clindamycin $(2 \mu \mathrm{g})$, vancomycin $(30 \mu \mathrm{g})$, teicoplanin $(30$ $\mu \mathrm{g})$, linezolid $(10 \mu \mathrm{g})$, tetracycline $(30 \mu \mathrm{g})$, ciprofloxacin $(5$ $\mu \mathrm{g})$, SXT (1.25 / $23.75 \mu \mathrm{g})$, ampicillin $(10 \mu \mathrm{g})$, AMC (20 / $10 \mu \mathrm{g})$, piperacillin $(30 \mu \mathrm{g})$, ceftriaxone $(30 \mu \mathrm{g})$, ceftazidime $(10 \mu \mathrm{g})$, cefepime $(30 \mu \mathrm{g})$, imipenem $(10 \mu \mathrm{g})$, and tigecycline $(15 \mu \mathrm{g})$ were placed on the isolated bacterial strains. After incubation at $37^{\circ} \mathrm{C}$ for $24 \mathrm{~h}$, zone diameters were measured and evaluated in accordance with the European Committee on Antimicrobial Susceptibility Testing.

For the honey samples, honey suspensions were prepared in two different concentrations. First, $50 \mu \mathrm{L}$ of untreated-pure honey samples were taken and 6-mm diameter antibiotic-free discs (Oxoid, UK) were added. These were kept under sterile conditions to enable the honey to be fully absorbed by the disc. Second, the honey samples were diluted with $50 \%$ distilled water in the same manner as after the impregnation and in $0.5 \mathrm{McFarland}$ turbidity standards. The bacterial suspension was then placed on the inoculated Mueller-Hinton agar using a sterile pen set. After incubation at $37^{\circ} \mathrm{C}$ for $24 \mathrm{~h}$, zone diameters were measured and evaluated (Rani, et al., 2017).

\section{Statistical Analysis}

Statistics were performed using SPSS (15.0). We applied a normality test (Shapiro-Wilk Test) to our data and the results were non-parametric. The Mann Whitney U Test was used to compare the chestnut and flower honey group variables $(\mathrm{P}<0.05$ or $\mathrm{P}<0.01)$. The Spearman Correlation Test was used to determine the correlation between the properties of the studied honey samples $(\mathrm{P}<0.05$ or $\mathrm{P}<0.01)$.

\section{Results}

\section{Melissopalynologycal Analysis}

We divided the honey samples into two main groups according to melissopalynologycal analysis: chestnut honey (chestnut pollen concentration $>70, \mathrm{n}=12$ ) (Terrab et.al., 2003; Turkish Food Codex, 2020) and flower honey $(\mathrm{n}=12)$ (Table 1).

\section{Physicochemical Analysis}

The physicochemical characteristics of the honey samples are summarized in Table 1 and 2. They were examined based on Codex Standards (CS) and Turkish Food Codex (TFC) Honey limits. 
Table 1. Location and melissopalynologycal analysis results of the studied honey samples.

\begin{tabular}{|c|c|c|c|c|c|}
\hline Sample Number & FSHS & Dominant $\mathrm{P}(>45 \%)$ & econdary P (16-449 & prtant Minor P (3-1 & Minor P $(<3 \%)$ \\
\hline$\overline{1}$ & \multirow{12}{*}{$\begin{array}{c}\text { Chestnut Honey } \\
\text { Samples }\end{array}$} & $\% 95$ Fagaceae & - & $\% 3$ Fabaceae & $\% 2$ Asteraceae \\
\hline 4 & & $\% 95$ Fagaceae & - & $\% 3$ Poaceae & $\% 2$ Lamiaceae \\
\hline 5 & & $\% 95$ Fagaceae & - & $\% 3$ Asteraceae & $\% 2$ Fabaceae \\
\hline 6 & & $\% 75$ Fagaceae & $\% 20$ Ericaceae & $\% 5$ Fabaceae & - \\
\hline 7 & & $\% 77$ Fagaceae & - & $\begin{array}{l}\% 13 \text { Poaceae } \\
\% 7 \text { Fabaceae } \\
\% 3 \text { Ericaceae }\end{array}$ & - \\
\hline 9 & & \%99 Fagaceae & - & \multirow[b]{2}{*}{$\begin{array}{l}\% 11 \text { Fabaceae } \\
\% 4 \text { Asteraceae }\end{array}$} & $\% 1$ Other \\
\hline 11 & & \%84 Fagaceae & - & & $\% 1$ Ericaceae \\
\hline 12 & & \%96 Fagaceae & - & $\% 4$ Fabaceae & - \\
\hline 13 & & $\% 95$ Fagaceae & - & \%5 Lamiacae & - \\
\hline 23 & & \%99 Fagaceae & - & - & $\% 1$ Other \\
\hline 19 & & $\% 92$ Fagaceae & - & $\% 8$ Poaceae & - \\
\hline 20 & & $\% 80$ Fagaceae & - & $\begin{array}{l}\% 10 \text { Fabaceae } \\
\% 5 \text { Asteraceae } \\
\% 5 \text { Apiaceae }\end{array}$ & - \\
\hline 2 & & \%70 Fabaceae & & $\begin{array}{c}\% 10 \text { Brassicaceae } \\
\% 5 \text { Poaceae } \\
\% 15 \text { Apiaceae }\end{array}$ & - \\
\hline 3 & & $\% 85$ Fabaceae & - & $\begin{array}{l}\% 8 \text { Poaceae } \\
\% 4 \text { Ericaceae }\end{array}$ & $\% 3$ Rosaceae \\
\hline 8 & & $\% 48$ Fabaceae & $\begin{array}{c}\% 20 \text { Asteraceae } \\
\% 17 \text { Fagaceae }\end{array}$ & $\begin{array}{l}\% 8 \text { Poaceae } \\
\% 5 \text { Ericaceae }\end{array}$ & $\% 2$ Lamiaceae \\
\hline 10 & & $\% 45$ Fagaceae & $\begin{array}{l}\% 35 \text { Fabaceae } \\
\% 20 \text { Ericaceae }\end{array}$ & - & - \\
\hline 14 & & $\% 57$ Fabaceae & $\% 17$ Asteraceae & $\begin{array}{l}\% 11 \text { Apiaceae } \\
\% 7 \text { Poaceae } \\
\% 4 \text { Araliaceae } \\
\% 4 \text { Tiliaceae }\end{array}$ & - \\
\hline 15 & $\begin{array}{l}\text { Flower Honey } \\
\text { Samples }\end{array}$ & $\% 62$ Ericaceae & $\% 27$ Fabaceae & $\begin{array}{l}\% 8 \text { Tiliacaeae } \\
\% 3 \text { Pinacaceae }\end{array}$ & - \\
\hline 16 & & - & $\begin{array}{l}\% 41 \text { Ericaceae } \\
\% 33 \text { Fabaceae }\end{array}$ & $\begin{array}{l}\% 12 \text { Fagaceae } \\
\% 6 \text { Tiliaceae } \\
\% 4 \text { Poaceae }\end{array}$ & $\begin{array}{c}\% 2 \text { Asteraceae } \\
\% 2 \text { Pinaceae }\end{array}$ \\
\hline 17 & & $\% 45$ Fagaceae & $\begin{array}{l}\% 36 \text { Ericaceae } \\
\% 19 \text { Fabaceae }\end{array}$ & - & - \\
\hline 18 & & $\% 50$ Fabaceae & $\% 25$ Tiliaceae & $\begin{array}{c}\% 15 \text { Fagaceae } \\
\% 6 \text { Ericaceae } \\
\% 4 \text { Asteraceae }\end{array}$ & - \\
\hline 21 & & $\% 62$ Fabaceae & - & $\begin{array}{l}\text { \%14 Asteraceae } \\
\% 11 \text { Rosaceae } \\
\% 9 \text { Lamiaceae } \\
\% 4 \text { Apiaceae }\end{array}$ & - \\
\hline 24 & & \%55 Lamiaceae & $\% 35$ Asteraceae & $\% 10$ Fabaceae & - \\
\hline
\end{tabular}

FSHS: Floral Source of Honey Samples, P: Pollen

\section{Total Phenolic Content and DPPH Radical Scavenging Activity (Antioxidant Activity)}

In the present study, the highest phenolic content (30.363 mg GAE / $100 \mathrm{~g}$ ) was determined in chestnut honey and lowest phenolic content (4.958 mg GAE / 100 g) was in flower honey sample. The total phenolic content was positively correlated $(\mathrm{P}<0.01)$ with the conductivity and color. In addition, significant differences were determined between the floral and chestnut honey groups on phenolic contents, chestnut honey group's phenolic content found higher than the flower honey group $(\mathrm{P}<0.01)$.

The DPPH radical removal activity was identified by the determination of the IC50 (half maximal inhibitory concentration) value (Table 4). The IC50 value is inversely proportional to the radical scavenging activity; thus, when the IC50 value increases, the radical scavenging activity decreases accordingly. The lowest IC50 (9.578 mg / mL) was determined on chestnut honey group and the highest IC50 value $68.002 \mathrm{mg} / \mathrm{mL}$ on the flower honey group. As expected, our data revealed a negative correlation $(\mathrm{P}<0.01)$ between IC50 value, total phenolic content, and color. We determined significant differences between the flower and chestnut honey groups on DPPH radical scavenging activity $(\mathrm{P}<0.01)$, which was found higher in the chestnut honey group. 
Table 2. Physicochemical analysis of chestnut honey samples. ("TFC=Turkish Food Codex Limits,2020)

\begin{tabular}{|c|c|c|c|c|c|c|c|c|c|c|c|c|c|c|}
\hline \multicolumn{15}{|c|}{ Chestnut Honey Samples } \\
\hline Sample Number & 1 & 4 & 5 & 6 & 7 & 9 & 11 & 12 & 13 & 23 & 19 & 20 & MEAN & $\overline{\mathrm{TFC}}$ \\
\hline Colour (mm) & 91 & 109 & 114 & 94 & 91 & 137 & 80 & 150 & 68 & 138 & 103 & 140 & $109.5 \pm 16.49$ & - \\
\hline Humidity (\%) & 18.3 & 21.2 & 21.2 & 18.4 & 20 & 18.6 & 18 & 19.6 & 16.5 & 22.1 & 18.8 & 17.7 & $19.20 \pm 1.65$ & $20(\mathrm{ma}$ \\
\hline HMF (mg/kg) & 20.2 & 10.9 & 11.5 & 6 & 27.8 & 5.4 & 40.1 & 3.4 & 20.7 & 9 & 14.4 & 14.6 & $15.3 \pm 10.55$ & $40(\max$ \\
\hline Diastase Number (DN) & 11.4 & 15.6 & 16.1 & 14.7 & 6.5 & 16.7 & 16.1 & 16.7 & 11.4 & 10.9 & 10.9 & 16.7 & $13.6 \pm 3.32$ & 8 (min) \\
\hline Invertase (U/kg) & 236.8 & 193.7 & 191.9 & 227.3 & 81.1 & 292.3 & 197.1 & 188 & 213.9 & 128 & 88.4 & 149.1 & $182.36 \pm 6.50$ & - \\
\hline Prolin (mg/kg) & 808.9 & 1001 & 1042. & 1033.5 & 106 & 600 & 122 & 66 & 98 & 3731 & 687 & 7709 & $879.59=$ & 500 (min) \\
\hline Acidity (m.q. g/kg) & 38 & 32 & 30 & 22 & 34 & 26 & 38 & 30 & 26 & 22 & 28 & 30 & $29.67 \pm 5.31$ & $50(\max )$ \\
\hline C. $(\mu \mathrm{S} / \mathrm{cm})\left(20^{\circ} \mathrm{C}\right)$ & 684 & 1508 & 1512 & 1591 & 965 & 1622 & 683 & 1953 & 215 & 2170 & 870 & 1752 & 1293.75 & - \\
\hline Fructose (\%) DIN & 38.8 & 37.1 & 38.2 & 37 & 37.3 & 34 & 38.1 & 32.8 & 38 & 35.5 & 35.4 & 36.2 & $36.53 \pm 1.82$ & - \\
\hline (\%) DIN & 19.6 & 22.1 & 22.8 & 19 & 26.8 & 16.7 & 27.1 & 16.4 & 26.5 & 20.5 & 25.4 & 19 & $21.83 \pm 3.89$ & - \\
\hline Fructose + Glucose $(\%)$ & 58.4 & 59.2 & 61 & 56 & 64.1 & 50.7 & 65.2 & 49.2 & 64.5 & 56 & 60.8 & 55.2 & $58.36 \pm 5.17$ & $60(\mathrm{mi}$ \\
\hline Fructose / Glucose (DIN) & 1.98 & 1.68 & 1.68 & 1.95 & 1.39 & 2.04 & 1.41 & 2 & 1.43 & 1.73 & 1.39 & 1.91 & $1.72 \pm 0.26$ & $1-1,85$ \\
\hline / Water (DIN) & 1.07 & 1.04 & 1.08 & 1.03 & 1.34 & 0.9 & 1.51 & 0.84 & 1.61 & 0.93 & 1.35 & 1.07 & $1.15 \pm 0.25$ & - \\
\hline Saccarose (\%) DIN & 0.3 & 0.1 & 0.1 & 0.1 & 0.1 & 0.2 & 0.2 & 0.2 & 0.1 & 0.1 & 0.2 & 0.2 & $0.16 \pm 0.06$ & $5(\max$ \\
\hline se (\%) DIN & 2.4 & 2 & 2 & 2.3 & 1.4 & 2.2 & 1.8 & 2.1 & 2.3 & 1.9 & 2.2 & 2.3 & $2.08 \pm 0.28$ & - \\
\hline Maltose (\%) DIN & 3 & 2.5 & 2.6 & 3.9 & 1.8 & 4.4 & 1.7 & 4 & 2.8 & 3.4 & 2.7 & 4.6 & $3.12 \pm 0.95$ & - \\
\hline ltose (\%) DIN & 1.1 & 0.5 & 0.5 & 1.3 & 0.3 & 1.8 & 0.5 & 2 & 0.6 & 0.9 & 0.7 & 1.5 & $0.98 \pm 0.56$ & - \\
\hline Erlose (\%) DIN & 0 & 0 & 0.2 & 0.1 & 0.1 & 0.4 & 0.1 & 0.1 & 0.3 & 0.1 & 0.1 & 0 & $0.13 \pm 0.12$ & - \\
\hline Melezitose (\%) I & 0.5 & 0.1 & 0.2 & 0.2 & 0.1 & 0.1 & 0.1 & 0.8 & 0.4 & 0.1 & 0.1 & 0.3 & $0.25 \pm 0.22$ & - \\
\hline Maltotriose (\%) DIN & 0.4 & 0.1 & 0 & 0 & 0.1 & 0 & 0.3 & 0 & 0.3 & 0 & 0.1 & 0.1 & $0.12 \pm 0.14$ & - \\
\hline
\end{tabular}

C.: Conductivity

Table 3. Physicochemical analysis of flower honey samples. (*TFC=Turkish Food Codex Limits,2020)

\begin{tabular}{|c|c|c|c|c|c|c|c|c|c|c|c|c|c|}
\hline \multicolumn{14}{|c|}{ Flower Honey Samples } \\
\hline Sample Number & 2 & 3 & 8 & 10 & 14 & 15 & 16 & 17 & 18 & 21 & 24 & MEAN & TFC \\
\hline Colour (mm) & 73 & 56 & 96 & 55 & 77 & 32 & 48 & 48 & 82 & 41 & 40 & $58.18 \pm 10.81$ & - \\
\hline Humidity (\%) & 16.5 & 20.5 & 16.6 & 18.2 & 17.8 & 17.7 & 17.8 & 17.8 & 16.3 & 16.4 & 18.6 & $17.69 \pm 1.24$ & $20(\max )$ \\
\hline HMF (mg/kg) & 19.3 & 17.5 & 100.2 & 29 & 59.1 & 19 & 18.4 & 18.4 & 10.6 & 32.8 & 38.2 & $33.43 \pm 5.81$ & $40(\max )$ \\
\hline Diastase Number (DN) & 14.3 & 13.9 & 9.8 & 14.3 & 5.9 & 7.8 & 5.9 & 5.9 & 11.4 & 7.8 & 7.5 & $9.67 \pm 3.28$ & $8(\min )$ \\
\hline Invertase (U/kg) & 188.5 & 196 & 38.3 & 153.2 & 61.7 & 73 & 250.2 & 250.2 & 270.2 & 71.2 & 7.8 & $131.75 \pm 8.85$ & - \\
\hline Prolin $(\mathrm{mg} / \mathrm{kg})$ & 600 & 683.7 & 596.3 & 856.6 & 755. & 329.9 & 455.2 & 155.2 & 76.4 & 566.5 & 683.7 & $602.77 \pm 14.13$ & 300 (min) \\
\hline Acidity (m.q. g/kg) & 40 & 34 & 28 & 32 & 28 & 18 & 30 & 30 & 30 & 20 & 24 & $27.82 \pm 6.48$ & $50(\max )$ \\
\hline C. $(\mu \mathrm{S} / \mathrm{cm})\left(20^{\circ} \mathrm{C}\right)$ & 812 & 528 & 459 & 431 & 433 & 525 & 536 & 536 & 683 & 248 & 231 & $488.55 \pm 17.04$ & - \\
\hline Fructose (\%) DIN & 27.8 & 36.1 & 35.2 & 36.4 & 40.6 & 37 & 37.5 & 38.4 & 34.2 & 36.7 & 39.3 & $36.29 \pm 3.35$ & - \\
\hline Glucose (\%) DIN & 20.3 & 27.4 & 30.8 & 26.6 & 23.8 & 26.2 & 29.4 & 27.7 & 27.1 & 29.1 & 32.8 & $27.38 \pm 3.37$ & - \\
\hline Fructose +Glucose $(\%)$ & 48.1 & 63.5 & 66 & 63 & 64.4 & 63.2 & 66.9 & 66.1 & 61.3 & 65.8 & 72.1 & $63.67 \pm 5.89$ & $60(\min )$ \\
\hline Fructose / Glucose (DIN) & 1.37 & 1.32 & 1.14 & 1.37 & 1.71 & 1.41 & 1.28 & 1.39 & 1.26 & 1.26 & 1.2 & $1.34 \pm 0.15$ & $0.9-1.4$ \\
\hline Glucose / Water (DIN) & 1.23 & 1.34 & 1.86 & 1.46 & 1.34 & 1.48 & 1.65 & 1.52 & 1.66 & 1.77 & 1.77 & $1.55 \pm 0.20$ & - \\
\hline Saccarose (\%) DIN & 0.1 & 0.2 & 0.4 & 0.2 & 0.4 & 0.1 & 0.1 & 0.1 & 0.2 & 0.5 & 0.5 & $0.25 \pm 0.16$ & $5(\max )$ \\
\hline Turanose (\%) DIN & 7.10 & 1.8 & 1.6 & 2.1 & 2.3 & 1.8 & 1.8 & 2.1 & 2 & 1.9 & 1 & $2.32 \pm 1.62$ & - \\
\hline Maltose (\%) DIN & 2.8 & 1.5 & 1.8 & 2.3 & 2.3 & 1.9 & 1.5 & 1.8 & 2.7 & 4.1 & 2.2 & $2.26 \pm 0.75$ & - \\
\hline Isomaltose (\%) DIN & 1.2 & 0.5 & 0.4 & 0.6 & 0.4 & 0.4 & 0.4 & 0.3 & 0.9 & 0.2 & 0.1 & $0.49 \pm 0.31$ & - \\
\hline Erlose (\%) DIN & 0 & 0 & 0 & 0.1 & 0.1 & 0.9 & 0.1 & 0.1 & 0 & 1.6 & 0.5 & $0.31 \pm 0.51$ & - \\
\hline Melezitose (\%) DIN & 0.3 & 0.1 & 0.1 & 0.7 & 0.2 & 0.5 & 0.1 & 0.3 & 0.7 & 1.3 & 0.1 & $0.40 \pm 0.38$ & - \\
\hline Maltotriose (\%) DIN & 0.3 & 0.2 & 0.2 & 0.4 & 0.3 & 0.5 & 0.2 & 0.2 & 0 & 0.1 & 0.2 & $0.24 \pm 0.14$ & - \\
\hline
\end{tabular}

C.: Conductivity

\section{Antimicrobial Properties}

It was seen that the pure honey samples in the study formed a larger zone diameter against the bacteria than the $50 \%$ dilution versions.

A. baumannii was resistant to all antibiotics, while only Tigecycline ( $\geq 19$ susceptible) showed a zone diameter of $15 \mathrm{~mm}$, although this was not within the limits of susceptibility. Considering this information, it was found that two chestnut honey samples formed a 13-mm zone diameter against the A. baumannii strain.

One sample form each honey groups formed a 22-mm and 21-mm zone against the MSSA strain. These values are larger than the zone diameters of penicillin, erythromycin, vancomycin and teicoplanin against the MSSA strain. Interestingly it was determined that honey samples having higher antimicrobial activity include lower moisture content, which means that when the moisture rate increased, antimicrobial activity of the studied pure honeys against MSSA decreased accordingly. Zone diameters were observed on eight chestnut honey samples and six flower honey sample against the MRSA strain. However, it was found that three chestnut honey samples formed the largest zone diameter $(20 \mathrm{~mm})$, which was wider than that of penicillin and erythromycin and similar to the vancomycin zone diameter (Tables 5 and 6). 
Table 4. Determination of total phenolic content and DPPH radical scavenging activity (antioxidant activity study) of honey samples.

\begin{tabular}{|c|c|c|c|}
\hline FSHS & Sample Number & Total Phenolic Content (mg GAE /100g Honey & Radical-scavenging activity (IC50) $\mathrm{mg} / \mathrm{ml}$ \\
\hline & $\mathrm{C} 1$ & 19.150 & 24.454 \\
\hline & 4 & 26.504 & 26.489 \\
\hline & 5 & 25.106 & 18.572 \\
\hline & 6 & 18.533 & 16.477 \\
\hline & 7 & 19.100 & 18.679 \\
\hline Chestnut Honey & 9 & 29.514 & 13.962 \\
\hline \multirow[t]{11}{*}{ Samples } & 11 & 12.197 & 30.298 \\
\hline & 12 & 27.109 & 13.803 \\
\hline & 13 & 9.925 & 34.416 \\
\hline & 23 & $30.363^{*}$ & 13.056 \\
\hline & 19 & 18.467 & 16.757 \\
\hline & 20 & 27.661 & $9.578^{*}$ \\
\hline & Mean & $21.97 \pm 6.72$ & $19.71 \pm 7.59$ \\
\hline & F2 & 11.605 & 33.137 \\
\hline & 3 & 10.604 & 48.374 \\
\hline & 8 & 11.154 & 27.572 \\
\hline & 10 & 7.140 & 48.892 \\
\hline \multirow{8}{*}{$\begin{array}{l}\text { Flower Honey } \\
\text { Samples }\end{array}$} & 14 & 9.444 & 32.067 \\
\hline & 15 & 4.958 & 51.423 \\
\hline & 16 & 6.453 & 56.657 \\
\hline & 17 & 6.198 & 48.754 \\
\hline & 18 & 10.331 & 25.283 \\
\hline & 21 & 7.302 & 68.002 \\
\hline & 24 & 5.916 & 56.341 \\
\hline & Mean & $8.28 \pm 2.38$ & $45.14 \pm 9.70$ \\
\hline
\end{tabular}

FSHS: Floral Source of Honey Samples, P: Pollen

Table 5. Inhibition zone diameters $(\mathrm{mm})$ of clinically important antibiotics against studied bacteria strains.

\begin{tabular}{l|cccc}
\hline \multirow{2}{*}{ Antibiotics } & \multicolumn{2}{c}{ S. aureus } & \multirow{2}{*}{ E. coli } & A. baumannii \\
\cline { 2 - 3 } & MSSA & MRSA & - & - \\
Penisilin $(1 \mu \mathrm{g})$ & 11 & 0 & - & - \\
Sefoksitin $(30 \mu \mathrm{g})$ & 30 & 10 & - & - \\
Eritromisin $(15 \mu \mathrm{g})$ & 14 & 26 & - & - \\
Klindamisin $(2 \mu \mathrm{g})$ & 25 & 20 & - & - \\
Vankomisin $(30 \mu \mathrm{g})$ & 20 & 18 & - & - \\
Teikoplanin $(30 \mu \mathrm{g})$ & 19 & 28 & - & - \\
Linezolid $(10 \mu \mathrm{g})$ & 28 & 28 & - & - \\
Tetrasiklin $(30 \mu \mathrm{g})$ & 27 & 28 & 34 & 0 \\
Siprofloksasin $(5 \mu \mathrm{g})$ & 25 & 24 & 22 & 0 \\
Gentamisin $(10 \mu \mathrm{g})$ & 24 & 32 & 30 & 0 \\
SXT $(1.25 / 23.75 \mu \mathrm{g})$ & 33 & - & 24 & 0 \\
Ampisilin $(10 \mu \mathrm{g})$ & - & - & 25 & 0 \\
AMC $(20 / 10 \mu \mathrm{g})$ & - & - & 28 & 0 \\
Piperasilin $(30 \mu \mathrm{g})$ & - & - & 27 & 0 \\
Seftriakson $(30 \mu \mathrm{g})$ & - & - & 24 & 0 \\
Seftazidim $(10 \mu \mathrm{g})$ & - & - & 34 & 0 \\
Sefepim $(30 \mu \mathrm{g})$ & - & - & 34 & 15 \\
İmipenem $(10 \mu \mathrm{g})$ & - & - & 21 & \\
Tigesiklin $(15 \mu \mathrm{g})$ & - & & & 0 \\
\hline
\end{tabular}

When we compared the honey groups, Chestnut honey samples tented to formed larger zone against studied bacteria strains than flower honey; but these differences did not show statistically significant distinction $\mathrm{P}>0.05$ ). According to physicochemical characteristics were isomaltose content was higher in chestnut honey samples. Additionally, Isomaltose content of honey samples was positively correlated with inhibition zone diameters, accordingly, antimicrobial effect on MRSA and A. baumanni $(\mathrm{P}<0.01)$

\section{Discussion}

Hospital-acquired infections (HAI) have usually acquired and manifest after hospitalization and showed and can cause serious complications (Boev and Kiss, 2017). Since increasing of the bacterial resistance to antibiotics become an important problem, scientists started to focus on natural products such as honey. 
Table 6. Inhibition zone diameters $(\mathrm{mm})$ of honey samples against studied bacteria strains.

\begin{tabular}{|c|c|c|c|c|c|c|c|c|c|}
\hline \multirow{3}{*}{ Honey types } & \multirow{3}{*}{ Sample Number } & \multicolumn{4}{|c|}{ S. aureus } & \multirow{2}{*}{\multicolumn{2}{|c|}{ E. coli }} & \multirow{2}{*}{\multicolumn{2}{|c|}{ A. baumannii }} \\
\hline & & \multicolumn{2}{|r|}{ MSSA } & \multicolumn{2}{|r|}{ MRSA } & & & & \\
\hline & & $\overline{\mathrm{PH}}$ & $\% 50$ dilüsyon & $\mathrm{PH}$ & $\% 50$ dilu & $\mathrm{PH}$ & $\% 50$ dilu & $\mathrm{PH}$ & $\% 50$ dilution \\
\hline \multirow{12}{*}{ Chestnut Honey Samples } & 1 & 11 & 7 & 0 & 0 & 0 & 0 & 0 & 0 \\
\hline & 4 & 0 & 0 & 0 & 0 & 0 & 0 & 0 & 0 \\
\hline & 5 & 0 & 0 & 0 & 0 & 0 & 0 & 0 & 0 \\
\hline & 6 & 13 & 10 & 18 & 11 & 0 & 0 & 13 & 8 \\
\hline & 7 & 13 & 8 & 12 & 0 & 15 & 0 & 0 & 0 \\
\hline & 9 & 21 & 15 & 20 & 12 & 14 & 8 & 10 & 8 \\
\hline & 11 & 20 & 18 & 20 & 9 & 15 & 0 & 8 & 0 \\
\hline & 12 & 22 & 20 & 18 & 9 & 13 & 0 & 8 & 0 \\
\hline & 13 & 22 & 11 & 16 & 12 & 0 & 0 & 13 & 9 \\
\hline & 19 & 16 & 9 & 15 & 10 & 0 & 0 & 7 & 0 \\
\hline & 20 & 21 & 10 & 20 & 10 & 0 & 0 & 7 & 0 \\
\hline & 23 & 12 & 0 & 0 & 0 & 0 & 0 & 0 & 0 \\
\hline \multirow{12}{*}{ Flower Honey Samples } & 2 & 21 & 17 & 15 & 13 & 0 & 0 & 9 & 8 \\
\hline & 3 & 0 & 0 & 11 & 0 & 0 & 0 & 0 & 0 \\
\hline & 8 & 10 & 8 & 0 & 0 & 13 & 0 & 10 & 0 \\
\hline & 10 & 22 & 10 & 15 & 9 & 14 & 0 & 11 & 0 \\
\hline & 14 & 20 & 15 & 14 & 13 & 0 & 0 & 0 & 0 \\
\hline & 15 & 14 & 0 & 0 & 0 & 0 & 0 & 0 & 0 \\
\hline & 16 & 14 & 12 & 9 & 0 & 0 & 0 & 0 & 0 \\
\hline & 17 & 14 & 10 & 0 & 0 & 0 & 0 & 0 & 0 \\
\hline & 18 & 20 & 14 & 15 & 13 & 0 & 0 & 0 & 0 \\
\hline & 21 & 15 & 0 & 0 & 0 & 0 & 0 & 0 & 0 \\
\hline & 22 & 0 & 0 & 0 & 0 & 0 & 0 & 0 & 0 \\
\hline & 24 & 11 & 0 & 0 & 0 & 0 & 0 & 0 & 0 \\
\hline
\end{tabular}

The most important factors which determine the therapeutic value of the honey are antimicrobial and antioxidant activities and both activities inflicting from honey's chemical contents (Gheldof, et al., 2002; Masalha et al., 2018; Aikaterini, 2019). In the current study we identified their antimicrobial and antioxidant activity of honeys which were identified the botanical origin and chemical contents. In this study we focused on chestnut and flower honey's effect on four of the common pathogens that are known to cause HAI; Escherichia coli, Staphylococcus aureus (MRSA-MSSA), Acinetobacter baumannii. (Young and Khadaroo 2014; Boev and Kiss, 2017). Two chestnut honey of studied samples showed similar zone diameter on A. baumannii strain as tigecycline which is the drug in the glycylcycline class of antibiotics. Chestnut honey samples tented to formed larger zone against studied bacteria strains than flower honeys, but these differences did not show statistically significant distinction. Also 5 chestnut honey samples showed wider zone than that of penicillin and erythromycin and similar to the vancomycin zone diameter on MRSA and MSSA but 4 flower honey samples showed the same performance only on MSSA strain. Consistent with our findings, Sherlock et al. (2010) reported a 17-24-mm zone diameter for Manuka honey which are well known around the world for their antimicrobial activity and one of 23-34 mm for Ulmo honey. Also, Kolaylı et al. (2020) determined antimicrobial activity of Turkish honey samples and four different Manuka honey samples; according to their findings Manuka honey samples did not show any inhibition zone for $E$. coli and showed $8 \mathrm{~mm}$ zone diameter for $S$. aureus while Chestnut honey samples showed $8 \mathrm{~mm}$ for $E$. coli and $10 \mathrm{~mm}$ for $S$. aureus. In our study five chestnut honey's inhibition zones for the MRSA strain were higher than that of Manuka honeys. In addition to botanical origin significant correlation was determined between antimicrobial activity and some chemical parameters. One of these parameters is Isomaltose. The amount of isomaltose and zone diameters of honeys for MRSA and A. baumannii showed linear correlation with each other. Isomaltose content appeared to be distinctively higher in chestnut honey samples. We determined Isomaltose content of chestnut honeys between; $2.0-0.3(\overline{\mathrm{x}}$ : $0,98)$ and in flower honeys 0.1-1.2 ( $\mathrm{x}: 0,49)$. Cotte, et al. (2003) determined the sugar profiles of some honeys and reported the average isomaltose percentage values of acacia, chestnut, and lavender honeys, respectively, as $0.89,1.80$, and $0.87 \%$. Also, Xu et al. 2020 shared some Japanese and Chinese commercial honey's isomaltose contents respectively between; 0,2-3,6 and 0-0,4. Mateo and Bosch-Reig (1997) detected isomaltose in some unifloral Spanish honeys in the range of $0.13-1.39 \%$. When we consider all the correlation data of our results, we can conclude that; the isomaltose contents were increased by the percentage of chestnut pollen in the honey. According to our results Isomaltose could be a new criterion for chestnut honey which is suitable in terms of honey standards' criteria. Recently in the literature, researchers focused on Isomaltose, for its biodegradability, antimicrobial activity, and boosting of intestinal microflora, as well as its being safely categorized as a prebiotic, precursor metabolite (Lee et al., 2008; Gerschenson, et al., 2017; Fatoki et al., 2018). Also, excitingly $\mathrm{Xu}$, et al. 2020 determined that; isomaltose content of honey contributes to the induction of 
granulocyte-colony stimulating factor (G-CSF) secretion in the intestinal epithelial cells following honey heating.

Another important factor which determines the therapeutic value of honey is antioxidant activity. The antioxidant activity of honey determined by its DPPH radical scavenging activity (Sharma and Bhat, 2009). Antioxidant activity of honey correlates with different properties of honey. Previous studies on different regional honey samples have shown that a positive correlation with phenolic content and the antioxidant activity of honey. (Beretta et al., 2005; Holderna Kedzia et al., 2006; Bertoncelj et al., 2007; Moniruzzaman et al., 2014; Nayik and Nanda, 2015; Çöl Ayvaz et al., 2018). These findings also consistent with ours. According to our results when the colour of the honey darkens, the phenolic content and connectedly antioxidant activity of honey increased. In our study most of the chestnut honey samples which has the highest phenolic content and darker color has shown the highest antioxidant activity.

When we consider all samples of the study, local chestnut and flower honeys of Yiğılca district showed no significant differences in terms of antimicrobial properties, although four flower and eight chestnut honeys from Yı̆̆ılca have higher antimicrobial activities than Manuka and other chestnut honeys from Turkey (Sherlock et al., 2010; Kolayli et. al., 2020). However, when antioxidant properties were evaluated, chestnut honeys showed higher antioxidant activities than flower honeys due to higher phenolic content. At this point, the health benefit index suggested by Masalha et al. (2018) could be a model for the future determination of the therapeutic values of the honey released to the market. The various types of honey, $\mathrm{CH}$ and $\mathrm{FH}$, demonstrated variable antioxidant and antibacterial activities. Types of $\mathrm{CH}$ were found to have a wide range of medicinal properties, probably due to their containing different nectar contents/phytochemicals. Additionally, we would like to emphasize two considerably overlooked parameters for the therapeutic honey samples. Under the light of these information, Isomaltose content for chestnut honey samples because of its antimicrobial activity and its potential to be a marker for chestnut honey quality. And invertase enzyme due to its sensitivity to heat and storage condition as a freshness indicator for both honey samples. These two parameters should be added to the codex as freshness and therapeutic value agents to classify and detect the medicinal value of honey.

\section{Acknowledgments}

This study was a part of scientific research titled "Investigation of the essential compositions, physicochemical and phenolic content, antimicrobial properties to determine medicine value of honey produced in Düzce and it was supported by Düzce University Scientific Research Fund (Project no. 2017.05.01.609). Authors also thanks to BEEO-Scientific Bio Solutions (SBS) LLC for physicochemical analysis support.

\section{References}

Aikaterini F. 2019. Therapeutic Properties of Honey. American Journal of Biomedical Science and Research. 4(6): 486-489. doi: 10.34297/ajbsr.2019.04.000858
Al Bshabshe A, Joseph MRP, Al Hussein A, Haimour W, Hamid ME. 2016. Multidrug resistance Acinetobacter species at the intensive care unit, Aseer Central Hospital, Saudi Arabia: A one year analysis. Asian Pacific Journal of Tropical Medicine, 9(9):903-908. doi: 10.1016/j.apjtm.2016.07.016

Almasaudi SB, Al-Nahari AAM, Abd El-Ghany ESM, Barbour E, Al Muhayawi SM, Al-Jaouni Azhar E, Qari M, Qari YA, Harakeh S. 2017. Antimicrobial effect of different types of honey on Staphylococcus aureus. Saudi Journal of Biological Sciences, 24(6): 1255-1261. doi: 10.1016/j.sjbs.2016.08.007

Basualdo C, Sgroy V, Finola MS, Juam M. 2007. Comparison of the antibacterial activity of honey from different provenance against bacteria usually isolated from skin wounds. Vet Microbiol, 124:375-381. doi: 10.1016/j.vetmic.2007.04.039

Bayram Y, Eren H, Berktas M. 2012. Distribution of bacterial pathogens in urine samples and resistance patterns of ESBL positive and negative Escherichia coli isolates against fosfomycin and other antimicrobials. Ankem Dergisi, 25(4): 232-236. doi: 10.5222/ankem.2011.232

Beretta G, Granata P, Ferrero M, Orioli M, Facino RM. 2005. Standardization of antioxidant properties of honey by a combination of spectrophotometric/fluorimetric assays and chemometrics. Analytica Chimica Acta, 533(2): 185-191. doi: 10.1016/j.aca.2004.11.010

Bertoncelj J, Doberšek U, Jamnik M, Golob T. 2007. Evaluation of the phenolic content, antioxidant activity and colour of Slovenian honey. Food Chemistry, 105(2): 822-828. doi: 10.1016/j.foodchem.2007.01.060

Boev C, Kiss E. 2017. Hospital-Acquired Infections: Current Trends and Prevention. Critical Care Nursing Clinics of North America, 29(1): 51-65. doi: 10.1016/j.cnc.2016.09. 012

Bogdanov S. 2009. International Honey Comission (IHC) International Honey Comission (IHC) - Harmonised methods of the international honey commission, 5: 1-62.

Bogdanov S, Lüllmann C, Martin P, Von Der Ohe W, Russmann H, Vorwohl G, Persano Oddo L, Sabatini AG, Luigi Marcazzan G, Piro R, Flamini G, Morlot M, Lhéritier J, Borneck Panagyotis R, Angelica M, Jacob Kerkvliet T, Ortiz A, Ivanov T, D'Arcy B, Mossel B, Vit P. 1999. International Honey Comission- Honey quality and international regulatory standards: review by the International Honey Commission, 80(2): 61-69.

Boussaid A, Chouaibi M, Rezig L, Hellal R, Donsì F, Ferrari G, Hamdi S. 2018. Physicochemical and bioactive properties of six honey samples from various floral origins from Tunisia. Arabian Journal of Chemistry, 11(2): 265-274. doi: 10.1016/j.arabjc.2014.08.011

Brand-Williams W, Cuvelier ME, Berset C. 1995. Respostas perceptivas E. LWT - Food Science and Technology, 28(1): 25-30. doi: 10.1016/S0023-6438(95)80008-5

Bravo L. 1998. Polyphenols: Chemistry, dietary sources, metabolism, and nutritional significance. Nutrition Reviews, 56(11): 317-33. doi: 10.1111/j.1753-4887.1998.tb01670.x.

Chauhan MS, Farooqui A, Trivedi A. 2017. Plants foraged by bees for honey production in northern India: The diverse flora of India and its implications for apiculture. Acta Palaeobotanica, 57(1): 119-132. doi: 10.1515/acpa-2017-0003

Chua LS, Rahaman NLA, Adnan NA, Tan TTE. 2013. Antioxidant activity of three honey samples in relation with their biochemical components. Journal of Analytical Methods in Chemistry, 2013: 1-8. doi: 10.1155/2013/313798

Cimpoiu C, Hosu A, Miclaus V, Puscas A. 2013. Determination of the floral origin of some Romanian honeys on the basis of physical and biochemical properties. Spectrochimica Acta Part A: Molecular and Biomolecular Spectroscopy, 100: 149154. doi: 1016/j.saa.2012.04.008

Codex Alimentations Draft revised standard for standard for honey (at step 10 of the Codex procedure) Alinorm. 2001, 01(25): 19-26. 
Çöl Ayvaz M, Ömür B, Ertürk Ö, Kabakçi D. 2018. Phenolic profiles, antioxidant, antimicrobial, and DNA damage inhibitory activities of chestnut honeys from Black Sea Region of Turkey. Journal of Food Biochemistry, 42(3): 110. doi: $10.1111 / \mathrm{jfbc} .12502$

Cotte JF, Casabianca H, Chardon S, Lheritier J, Grenier-Loustalot MF. 2003. Application of carbohydrate analysis to verify honey authenticity. Journal of Chromatography A, 1021(12): 145-155. doi: 10.1016/j.chroma.2003.09.005

Crane E. 1997. The past and present importance of bee products to man. In A. Mizrahi and Y. Lensky (Eds.), Bee products. Properties, applications, and apitherapy, pp:1-13. New York, NY: Springer Science+Business. doi: 10.1007/978-1-47579371-0_1

Derebaşı E, Bulut G, Col M, Güney F, Yaşar N, Ertürk Ö. 2014 Physicochemical and residue analysis of honey from Black Sea Region of Turkey. Fresenius Environmental Bulletin, 23(1): 10-17.

Duisberg H, Hadorn H. 1966. Welche Anforderungen sind an Handelshonige zu stellen? Mitt. Lebensm. and Hyg, 57: 386407.

Estevinho L, Pereira AP, Moreira L, Dias LG, Pereira E. 2008. Antioxidant and antimicrobial effects of phenolic compounds extracts of Northeast Portugal honey. Food and Chemical Toxicology, 46(12): 3774-3779. doi: 10.1016/j.fct.2008.09. 062

Frankel S, Robinson GE, Berenbaum MR. 1998. Antioxidant capacity and correlated characteristics of 14 unifloral honeys. Journal of Apicultural Research, 37(1): 27-31. doi: 1080/00218839.1998.11100951

Gerschenson LN, Rojas AM, Fissore EN. 2017. Carbohydrates. Nutraceutical and Functional Food Components, Academic Press, 39-101. doi: 10.1016/B978-0-12-805257-0.00003-X

Günal N. 2013. Türkiye'de iklimin doğal bitki örtüsü üzerindeki etkileri. Acta Turcica, 1: 1-22.

Hezekiah Fatoki T, Morakinyo Sanni D, Uwaremhevho Momodu D, Unuagbon Ugboko H, Joke Adeseko C, Christianah Faleye B. 2018. Evaluation of Empirical Functions and Fate of Isomaltose, Journal of Applied Life Sciences International, 16(2): $1-10$

Hołderna-Kędzia E, Kędzia B. 2006. Research on an antioxidant capacity of honeys. Acta Agrobotanica, 59(1): 265-269. doi: 10.5586/aa.2006.027

Kambur M, Kekeçoğlu M, Yildiz İ. 2015. Assesment of the Honey Samples Produced in Yığılca District of Düzce City by Using Chemical and Palynological Analysis. Uludag Bee Journal, 15(2): 67-79.

Kolaylı S, Palabıyık I, Atik DS, Keskin M, Bozdeveci A, Karaoğlu SA. 2020. Comparison of Antibacterial and Antifungal Effects of Different Varieties of Honey and Propolis Samples. Acta Alimentaria, 49(4): 515-523. doi: 10.1556/066.2020.49.4.18

Kuropatnicki AK, Kłósek M, Kucharzewski M. 2018. Honey as medicine: historical perspectives, Journal of Apicultural Research, 57(1): 13-118. doi:10.1080/00218839.2017.1411 182

Lee HC, Kim JH, Kim SY, Lee JK. 2008. Isomaltose Production by Modification of the Fructose-Binding Site on the Basis of the Predicted Structure of Sucrose Isomerase from "Protaminobacter rubrum." Applied and Environmental Microbiology, 74(16): 5183-5194. doi: 10.1128/aem.0018108

Liu JR, Ye YL, Lin TY, Wang YW, Peng CC. 2012. Effect of floral sources on the antioxidant, antimicrobial, and antiinflammatory activities of honeys in Taiwan. Food Chemistry, 139(1): 4938-943. PMid:23561193.

Lobo V, Patil A, Phatak A, Chandra N. 2010. Free radicals, antioxidants and functional foods: Impact on human health Pharmacognosy Reviews, 4(8): 118-126. doi:10.4103/09737847.70902
Louveaux J, Maurizio A, Vorwohl G. 1970. Internationale kommission für bienenbotanik Der I. U. B. S. Methodik der melıssopalynologie. Apidologie, 1: 193-209.

Machado De-Melo AA, Almeida-Muradian LB, De Sancho MT, Pascual-Maté A. 2018. Composición y propiedades de la miel de Apis mellifera: una revisión. Journal of Apicultural Research, 57(1): 5-37. doi: 10.1080/00218839.2017.1338 444

Masalha M, Abu-Lafi S, Abu-Farich B, Rayan M, Issa N, Zeidan M, Rayan AA. 2018. New Approach for Indexing Honey for Its Heath/Medicinal Benefits: Visualization of the Concept by Indexing Based on Antioxidant and Antibacterial Activities. Medicines, 5(4):135. doi: 10.3390/medicines5040135

Mateo R, Bosch-Reig F. 1997. Sugar profiles of Spanish unifloral honeys. Food Chemistry, 60(1): 33-41. doi: 10.1016/S03088146(96)00297-X

Maurizio A, Hodges FED. 2015. Pollen Analysis of Honey. Bee World, 32(1): 1-5. doi: 10.1080/0005772x.1951.11094660.

Moniruzzaman M, Yung An C, Rao PV, Hawlader MNI, Azlan SABM, Sulaiman SA, Gan SH. 2014. Identification of phenolic acids and flavonoids in monofloral honey from Bangladesh by high performance liquid chromatography: Determination of Antioxidant Capacity. BioMed Research International, 1-11. doi: 10.1155/2014/737490.

Nayik GA, Nanda V. 2015. Physico-chemical, enzymatic, mineral and colour characterization of three different varieties of honeys from Kashmir valley of India with a multivariate approach. Polish Journal of Food and Nutrition Sciences, 65(2): 101-108. doi: 10.1515/pjfns-2015-0022

Nimse SB, Palb D. 2015. Free radicals, natural antioxidants, and their reaction mechanisms. The Royal Society of Chemistry, 5: 27986-28006. DOI: 10.1039/c4ra13315c.

Oddo LP, Piazza MG, Pulcini P. 1999. Invertase activity in honey. Apidologie, 30(1): 57-65. doi: 10.1051/apido: 19990107

Ömür B. 2015. Karadeniz Bölgesinde üretilen kestane (Castanea sativa Mill.) ballarının biyokimyasal özelliklerinin incelenmesi. Master Thesis. T.C. Ordu Üniversitesi Fen Bilimleri Enstitüsü.

Özkök A. 2018. Türkiye'de hızla büyüyen sektör: Arı ürünlerine genel bir bakış (1. Bask1), Hacettepe Üniversitesi, Ankara: Palme Yayınları, ISBN: 978-975-491-463-4. pp. s.208.

Rani GN, Budumuru R, Bandaru NR. 2017. Antimicrobial activity of honey with special reference to methicillin resistant staphylococcus aureus (MRSA) and methicillin sensitive staphylococcus aureus (MSSA). Journal of Clinical and Diagnostic Research, 11(8): DC05-DC08. doi: 10.7860/JCDR/2017/30085.10347. 47.

Sharma OP, Bhat TK. 2009. DPPH antioxidant assay revisited. Food Chemistry, 113(4): 1202-1205. doi: 10.1016/j. foodchem.2008.08.008

Sherlock O, Dolan A, Power A, Gethin G, Cowman S, Humphreys. 2010. Comparison of the antimicrobial activity of Ulmo honey from Chile and Manuka honey against methicillin-resistant Staphylococcus aureus, Escherichia coli and Pseudomonas aeruginosa. BMC Complementary and Alternative Medicine, 10(1): 2-5. Doi: 10.1186/1472-6882-10-47.

Taormina PJ, Niemira BA, Beuchat LR. 2001. Inhibitory activity of honey against foodborne pathogens as influenced by the presence of hydrogen peroxide and level of antioxidant power. International Journal of Food Microbiology, 69(3): 217-225. doi: 10.1016/S0168-1605(01)00505-0

Terrab A, González AG, Díez MJ, Heredia FJ. 2003. Characterisation of Moroccan unifloral honeys using multivariate analysis. European Food Research and Technology, 218(1): 88-95. doi: 10.1007/s00217-003-0797-x

Turkish Food Codex Honey Communique (Türk Gida Kodeksi Bal Tebliği). 2020. (TEBLİ̆G NO: 2020/7) https://www.resmigazete.gov.tr/eskiler/2020/04/2020042213.htmhttp://www.resmigazete.gov.tr/eskiler/2012/07/20120 727-12.htm 
Valentini A, Miquel C, Taberlet P. 2010. DNA barcoding for honey biodiversity. Diversity, 2(4): 610-617. doi: $10.3390 / \mathrm{d} 2040610$.

Winkler O. 1955. Beitrag zum nachweis und zur bestimmung von oxymethylfurfural in Honig und Kunsthonig. Z. Lebensm.Unters. Forsch, 102: 160-167. 54.
$\mathrm{Xu}$ X, et.al. 2020. Honey isomaltose contributes to the induction of granulocyte-colony stimulating factor (G-CSF) secretion in the intestinal epithelial cells following honey heating. Scientific Reports, 10(1): 1-14. doi: 10.1038/s41598-02071993-w

Young IS, Woodside JV. 2001. Antioxidants in health and disease. Journal of Clinical Pathology, 54: 176- 186. doi: 10.1136/jcp.54.3.176

Young PY, Khadaroo RG. 2014. Surgical site infections. Surgical Clinics of North America, 94(6): 1245-1264. doi: 10.1016/j.suc.2014.08.008 